\title{
EDITORIAL
}

\section{Value of CVP: an epidemiological or physiological question?}

\author{
S. Magder
}

๑ 2016 Springer-Verlag Berlin Heidelberg and ESICM

Original investigations, reviews and meta-analysis repeatedly declare that central venous pressure (CVP) is not a useful measure. These discussions usually do not include the obvious diagnostic value. For example, if a resident makes a diagnosis of a pulmonary embolism to explain a patient's shock and then tells me that the CVP is zero, my response would be yes, that could happen, but you had better come up with some other creative processes to explain shock due to right heart obstruction and without venous back-up. Or the converse, the resident says that the shock is due to massive blood loss and the CVP is $20 \mathrm{mmHg}$. Again, I would require some creative pathophysiology.

Most often, "not useful" means that CVP does not indicate blood volume or predict whether cardiac output will increase with a fluid bolus. From a physiological perspective the potential of CVP to predict blood volume should not even be considered. CVP is determined by the interaction of cardiac function and the function determining the return of blood to the heart [1]. A change in either changes CVP with a constant blood volume. CVP is also normally close to zero and not so much a determinant of cardiac output but a consequence [2]. What CVP does is give an indication of how cardiac and return functions are interacting [3].

CVP as a predictor of volume responsiveness is a more difficult issue, and the subject of the paper by Eskesen et al. [4]. These authors hypothesized that if they examined individual patient data sets from the large number of studies that have examined fluid responsiveness, they could find a value of CVP below which fluid responsiveness is likely. However, after a lot of work and many sophisticated analyses they found no value of CVP useful for this purpose. Intuitively, this result does not seem

\footnotetext{
*Correspondence: sheldon.magder@muhc.mcgill.ca

Department of Critical Care, McGill University Heath Centre (Glen Site Campus), 1001 Decarie Blvd, room D03.6358, Montreal, QC H4A 3J1,
} Canada

\section{空 Springer}

to make sense. Which clinician would not think twice before giving a fluid bolus to a patient with undifferentiated hypotensive shock and a CVP of $5 \mathrm{mmHg}$, or at the other end of the spectrum, not pause before giving a bolus to a patient in shock and a CVP of $20 \mathrm{mmHg}$ ? For the record, in our study, which is included in the metaanalysis of Eskesen et al. [4], we found a clear gradient in fluid responsiveness going from $75 \%$ at $0-5 \mathrm{mmHg}$, $55 \%$ at $6-10 \mathrm{mmHg}, 15 \%$ from 10 to $14 \mathrm{mmHg}$, with no patient responding above $13 \mathrm{mmHg}$ [5]. To my mind the problem encountered by Eskesen et al. [4] is that they used an epidemiological tool to examine a physiological question. Furthermore, the epidemiological tool was applied to studies with many potential technical problems and an important bias.

Technical details are very important for any study on CVP because the range of values influencing cardiac output is very small. How carefully were transducers leveled? What was the reference level? (In our study transducers were leveled at $5 \mathrm{~cm}$ below the sternal angle, which means a pressure of $0 \mathrm{mmHg}$ would be $3 \mathrm{mmHg}$ if leveled at the mid-axillary position [5]). Where was the level on the transducer? Was mean pressure used or a value at end-expiration? Was active expiration ruled out? Where on the tracings were the measurements made? How fast was fluid given? How long did investigators wait to measure cardiac output? Another variable is the baseline cardiac output. If the plateau of cardiac function occurs at $5 \mathrm{~L} / \mathrm{min}$, a $1-\mathrm{mmHg}$ change in CVP will produce an approximately $0.5 \mathrm{~L} / \mathrm{min}$ change in cardiac output when on the ascending part of the cardiac function curve, but at peak exercise and a cardiac output of $25 \mathrm{~L} / \mathrm{min}$, a 1-mmHg change in CVP will increase cardiac output by $2.5 \mathrm{~L} / \mathrm{min}$. Eskesen et al. [4] performed secondary analyses of these confounders, but the power of these analyses was limited. Overall, the large sample just managed to increase background noise. 
The bigger problem, however, is that the study design in all of the studies in this meta-analysis by Eskesen et al. [4] -including our own [5] — have an important bias. We had progressively fewer boluses as CVP increased, and we had trouble obtaining values above $10 \mathrm{mmHg}$ [5]. The reason for this is that in all of these studies bedside clinicians make the decision to give the bolus. The clinicians have a pretest probability of the likelihood of response in their heads, and the data are not random but simply observational. Causal reasoning can, therefore, not be applied. A proper study of this question requires a physiological-and not an epidemiological-approach. A large sample of patients with CVP ranging from 0 to $20 \mathrm{mmHg}$ should be stratified, with equal numbers of subjects in different quartiles. They should be given sufficient volume to increase the CVP by $2 \mathrm{mmHg}$ to ensure that Starling's law is tested. Cardiac output should then be tested in a reliable and precise way within 15 min after the infusion. Of course, such a study cannot be conducted for both practical and the obvious ethical reasons. It cannot even be conducted in normal subjects because they do not reach sufficiently high CVP values unless given dangerous amounts of fluids rapidly.

The true question is: Do we really need this kind of study anyways? I believe we are asking the wrong question of the CVP. CVP needs to be integrated into a complete clinical approach to the management of the patient. This includes the initial analysis of the clinical problem and the potential for fluid to help. Ideally, this assessment should include a flow measurement, but if not, a surrogate of flow. More importantly, CVP should be included in what I call a "responsive" protocol in which one asks whether the bolus fixed the problem [3].

The paper by Eskesen et al. [4] sends both scientific and clinical messages. On the scientific side, accumulating individual data from multiple heterogeneous studies with different technical approaches, quality of measurements, and patient characteristics is unlikely to allow the researchers to arrive at a conclusion much different from that of each of the original studies. This is especially so when the studies have a common uncontrolled component, which in this case is the clinician's judgement. It should be clear that a CVP measurement is not the start of the decision-making process. CVP gives an indication of how well the heart is interacting with the return of blood. This has mechanistic and diagnostic value. The initial step is identification of a clinical problem that might respond to fluids. If CVP is low, an initial moderate fluid bolus is unlikely to cause harm, whereas when CVP is high, giving volume can compromise organ function. A high CVP indicates that the heart is not handling the venous return, and the clinical problem is likely complex. The most important step is the next one. After giving fluid, the clinician must determine what has happened. Was the trigger for fluid corrected or at least partially corrected? If not, was sufficient fluid given to test the Starling hypothesis? In that capacity CVP can be very helpful by indicating whether enough fluid was given and how the heart responded. I fully agree that a single value of CVP taken out of context of the clinical situation is not helpful. That is why we have physicians-and not computers-to manage patients.

\section{Compliance with ethical standards}

Conflicts of interest

The author has no conflict of interest to declare for this paper.

Received: 23 December 2015 Accepted: 29 December 2015

Published online: 27 January 2016

\section{References}

1. Guyton AC (1955) Determination of cardiac output by equating venous return curves with cardiac response curves. Physiol Rev 35:123-129

2. Magder S (2015) Understanding central venous pressure: not a preload index? Curr Opin Crit Care 21(5):369-375

3. Magder S (2012) An approach to hemodynamic monitoring: Guyton at the bedside. Crit Care 16:236-243

4. Eskesen TG, Wetterslev M, Perner A (2015) Systematic review including re-analyses of 1148 individual data sets of central venous pressure as a predictor of fluid responsiveness. Intensive Care Med. doi:10.1007/ s00134-015-4168-4

5. Magder S, Bafaqeeh F (2007) The clinical role of central venous pressure measurements. J Intensive Care Med 22(1):44-51 